\title{
Population and habitat responses to the global climate change in a widespread species, the Asiatic Toad (Bufo gargarizans)
}

\author{
Liuyang Yang ${ }^{1}$, Huabin Zhang ${ }^{1}$, Naijing Zhang ${ }^{1}$, Ruiqing Cai ${ }^{1}$, Peng Yan $^{2}$, En Li $^{1}$, Tao \\ $\mathrm{Pan}^{1}$, and Xiaobing $\mathrm{Wu}^{1}$ \\ ${ }^{1}$ Affiliation not available \\ ${ }^{2}$ Anhui Normal University
}

November 12, 2020

\begin{abstract}
Abstract: Global climate change is gradually changing the distribution and diversity pattern of species. In order to study the change of distribution area and distribution barycenter of Bufo gargarizans under climate change, we adopted the maximumentropy modeling (Maxent) model and barycenter migration analysis. And four General circulation models (GCMs) under four representative concentration pathways (RCPs), 29 environment variables (including bioclimate, topography, habitat and human impact) were used to predict the current and future (2050 and 2070) distribution of B. gargarizans. The results show that the Mean temperature of Driest quarter (Bio9), Precipitation of driest month (Bio14) and population (POP) have greater influence on the distribution of B. gargarizans, it indicates that climate and anthropogenic factors have greater influence on the distribution of B. gargarizans than other environmental factors such as topography and landform. And in the next 30 to 50 years, from the perspective of the large spatial scale of the whole China, the distribution area of B. gargarizans is in the eastern part of China, the southeastern part is reduced, and the Midwest and northwest part is expanded. On the whole, the suitable habitat of B. gargarizans will be reduced in the future and will migrate to the high latitude and altitude area. In this study, B. gargarizans was used as a sentinel species to study the response of amphibians to climate change, this paper aims to reveal the mechanism of amphibian response to climate change from the current and future dispersal patterns of B. gargarizans.
\end{abstract}

Population and habitat responses to the global climate change in a widespread species, the Asiatic Toad (Bufo gargarizans)

Liuyang Yang ${ }^{\mathrm{a}, \mathrm{d}} \mathbb{I}$, Huabin Zhang,c, $\mathbb{I}$, Naijing Zhang ${ }^{\mathrm{a}}$, Ruiqing Cai ${ }^{\mathrm{a}}$, Peng Yan ${ }^{\mathrm{a}}$, En Li ${ }^{*}$, Tao Pan $^{\text {* }}$, Xiaobing $\mathrm{Wu}^{\mathrm{a}^{*}}$

${ }^{a}$ Anhui Province Key Laboratory of the Conservation and Exploitation of Biological Resources, College of Life Sciences, Anhui Normal University, Wuhu 241000, Anhui, China;

$\mathbb{I}$ These authors contributed equally to this work.

* Author for correspondence: Xiaobing Wu, wuxb@ahnu.edu.cn; Tao Pan, pantao@ahnu.edu.cn; En Li, lien1971@mail.ahnu.edu.cn.

Address of corresponding author (they have the same address): Zhe Shan Campus, Anhui Normal University, Jinghu District, Wuhu city, Anhui Province, China.

Running Head: The Asiatic Toad respond to the climate change

Abstract:Global climate change is gradually changing the distribution and diversity pattern of species. In order to study the change of distribution area and distribution barycenter ofBufo gargarizans under climate change, we adopted the maximum-entropy modeling (Maxent) model and barycenter migration 
analysis. And four General circulation models (GCMs) under four representative concentration pathways (RCPs), 29 environment variables (including bioclimate, topography, habitat and human impact) were used to predict the current and future (2050 and 2070) distribution of B. gargarizans. The results show that the Mean temperature of Driest quarter (Bio9), Precipitation of driest month (Bio14) and population (POP) have greater influence on the distribution of $B$. gargarizans, it indicates that climate and anthropogenic factors have greater influence on the distribution of B. gargarizans than other environmental factors such as topography and landform. And in the next 30 to 50 years, from the perspective of the large spatial scale of the whole China, the distribution area of B. gargarizans is in the eastern part of China, the southeastern part is reduced, and the Midwest and northwest part is expanded. On the whole, the suitable habitat of $B$. gargarizans will be reduced in the future and will migrate to the high latitude and altitude area. In this study, B. gargarizans was used as a sentinel species to study the response of amphibians to climate change, this paper aims to reveal the mechanism of amphibian response to climate change from the current and future dispersal patterns of $B$. gargarizans .

Key words: Bufo gargarizans, Maxent, distribution, barycenter migration, climate change, sentinel species

\section{Introduction}

The distribution and interaction of species are closely related to climate (Nogues-Bravo \& Rahbek, 2011), and global climate change is a major challenge facing mankind and the biological world in the 21st century (Bertrand et al., 2011). According to the Intergovernment Panel on Climate Change (IPCC), average global temperatures have risen by about 1.5 compared with pre-industrial levels. And the rising global average temperature poses a serious threat to the sustainability of the global ecosystem and alters global biodiversity patterns (Dawson, Jackson, House, Prentice, \& Mace, 2011). If species can find niches to satisfy their needs, their habitats will expand or shrink as the climate changes. However, if climate-friendly habitats disappear or are affected by landscape barriers, some less diffuse species will become extinct as a result of climate warming.

Amphibians are at higher risk of extinction than other groups of vertebrates(Stuart et al., 2004; Wake \& Vredenburg, 2008), with 41 percent at risk, and due to their complex life history and unique physiological structure, their distribution and diffusion are greatly affected by climate and landscape, and their ability to cope with climate change is weak, which may be one of the reasons for the decline of the global amphibians population (Lambers, 2015; Sinervo et al., 2010).

In order to study the response of species to climate change, SDMs (SDMs) is usually chosen to simulate the distribution area of species. SDMs can calculate the relationship between the existence point of species and local climate variables, perform function fitting through different algorithms, and then project the function to a specific research area and time, and finally obtain the distribution of the target species' adaptive area (Elith, 2009; Guisan \& Thuiller, 2010). Although the SDMs has some shortcomings, for example, it cannot fully take into account the interactions between species (Pearson \& Dawson, 2003), SDMs is still one of the effective methods for predicting species distribution, assessing the risk of species extinction, planning and construction of protected areas (Li et al., 2014; Preston, Rotenberry, Redak, \& Allen, 2010). SDMs are often used to predict the distribution of endangered or narrow species, and there are relatively few studies on the prediction of widespread species (Yang, Tang, \& Luo, 2020).

B. gargarizans is one of the most widely distributed amphibians in China, and compared with other amphibians, it has a relatively low sensitivity to environmental factors, so it is a great sentinel species for revealing amphibian responses to temperature changes in this study. And the study of the genealogical geography of $B$. gargarizans shows that the differentiation of the subspecies of $B$. gargarizans is mainly caused by dominant dispersion rather than vicariance, and showed a trend of dispersion from west to east (Fu, Weadick, Zeng, Wang, \& Hu, 2005; Hu et al., 2007). Relevant studies show that in the future, the ecological niche of a large number of amphibians in China will migrate to the west or north, and the fragmentation of the distribution area will be serious year by year. (Duan, Kong, Huang, Varela, \& Ji, 2016). Although the genealogical geography of B. gargarizans is relatively thorough, its spatial distribution prediction has not been carried 
out in depth. Starting from the distribution area of B. gargarizans, this study aims to clarify the current and future trend of the $B$. gargarizans 's dispersion under the context of climate change, so as to provide certain data support for the response model of amphibians to climate change.

The Maxent model was selected to simulate the current and future spatial distribution of B. gargarizans under the background of global climate change and the migration of the centroid point, in order to solve the following problems: i) to simulate the current and future distribution of B. gargarizans in China; ii) to predict the change of suitable habitat of $B$. gargarizans in different parts of China under climate change; iii) to predict the distribution barycenter migration of $B$. gargarizans in China under climate change, and reveal the mechanism of amphibian response to climate change.

\section{Materials and methods}

\subsection{Occurrence records of $B$. gargarizans.}

The distribution data of $B$. gargarizans used in this study were mainly from two sources: i) Data accumulated through years of laboratory fieldwork, 69 records were recorded; ii) and were obtained from the Global Biodiversity Information Facility (GBIF), 287 records were obtained. GBIF is a global scientific organisation that specialises in providing basic data on biodiversity, the data is obtained by searching for the keyword $B$. gargarizans (visited in June 2020) (Jiang, Zhang, Gao, Cai, \& Zhang, 2019). Distance between all species distribution data $>1 \mathrm{Km}$ by processing in ArcGis10.2 software. Finally, there are 274 pieces of data for this study (Table S1).

\subsection{Environmental variables}

We collected 29 environmental variables for this study, which can be roughly divided into four categories: bioclimate, topography, habitat, and human impact, source of all variables show in Table S2. In order to avoid model overfitting, all collected variables were processed by the band collection statistics function in ArcGIS 10.2. Remove the environment variables with high correlation $(r>0.7)$. After screening, Bio3, Bio5, Bio9, Bio14, Bio15, slope, Veg, NDVI, POP, GDP and HI were used for this study. Bio3, Bio5, Bio9, Bio14, Bio15, slope, Veg, NDVI, POP, GDP and HI. Since variables other than future bioclimatic factors cannot be predicted and change relatively little over a short period of time, we assume that they remain constant and use their projections of the future distribution of B. gargarizans (Yang et al., 2020).

In order to reduce the uncertainty of model prediction, we chose four internationally recognized GCMs (BCCCSM1-1, HadGEM2-ES, IPSL-CM5A-LR, MIROC5) and four RCPs (RCP2.6, 4.5, 6.0 and 8.0) published in the IPCC fifth Assessment report for prediction in 2050 and 2070.

\section{3 Model optimization and parameter setting}

To establish the most stable and reliable model, we choose model calibration, which includes regularization multiplier (0.5 to 6 , interval 0.5) and combination of basic feature classes and from one different sets of all layers. The best parameters were selected based on statistical significance (partial ROC), predictive ability (miss rate $\mathrm{E}=5 \%$ ) and complexity level (AICc) by useing $\mathrm{R}$ package kuenm (Cobos, Peterson, Barve, \& Osorio-Olvera, 2019). After the model parameters were determined, chose the data output format of mathematical logic to ensure that the output data was between 0 and 1 . And the jackknife method was selected to evaluate the contribution of environmental variables. Then the distribution data ofB.gargarizans were randomly divided into two groups: $25 \%$ were randomly selected as the test set, and the remaining $75 \%$ were used as the training set, repeat Bootstrap replicates 10 times in MaxEnt.3.4.1. Other model parameters are selected by system default. Finally, the predictive performance of the model was validated by using the area under the receiver-operating characteristic (ROC) curve (AUC) and the mean omission error. The higher the AUC value, the more accurate the model performance. Normally, AUC $>0.9$ represents excellent prediction performance of the model. The ensemble threshold of model was calculated according to the Maximizing Sensitivity and Specificity (MSS) by using the dismo package in R. The MSS method is commonly used in presence only kind of occurrence data(Hijmans, Phillips, Leathwick, \& Elith, 2017; Liu, White, Newell, \& Pearson, 2013). 


\subsection{Distribution barycenter migration under different scenarios of future global climate change}

We divided the study area into small grids of $0.1 \operatorname{deg} x 0.1 \mathrm{deg}$ to evaluate the distribution barycenter migration of B. gargarizans in China. Assuming that our research area is composed of $\mathrm{N}$ small grids, the proportion of the $\mathrm{k}^{\text {th }}$ grid is $\mathrm{G}_{\mathrm{K}}=\mathrm{P}_{\mathrm{K}} \mathrm{x} \mathrm{S}_{\mathrm{K}}$, and $\mathrm{P}_{\mathrm{K}}$ represents the probability of the species appearing in the $\mathrm{k}^{\text {th }}$ small grid, and $\mathrm{S}_{\mathrm{K}}$ represents the area of the $\mathrm{k}^{\text {th }}$ small grid. The coordinates of each grid are obtained by Arcgis10.2. The following formula is used to obtain the coordinates of the barycenter:

$\mathrm{X}=\frac{\sum_{k=1}^{k} G_{k} X_{k}}{\sum_{k=1}^{k} G_{k}} ; \mathrm{Y}=\frac{\sum_{k=1}^{k} G_{k} Y_{k}}{\sum_{k=1}^{k} G_{k}}$

Here, $\mathrm{X}$ and $\mathrm{Y}$ represent the longitude and latitude of the $\mathrm{k}^{\text {th }}$ grid, respectively(He et al., 2011).

The results of four GCMs under four RCPS were averaged to obtain $\mathrm{P}_{\mathrm{f}}$, using ${ }^{P}\left({ }^{P}=P_{\mathrm{f}}-\right.$ $P_{\mathrm{c}}, P_{\mathrm{c}}$ representsthecurrentdistributionprobabilityof $B$. gargarizans)toevaluatethechangetrendof $B$. gargarizansdistribution $-0.1<D P[?] 0.1$ indicatedthatthehabitatsuitabilitychangeswerenotobvious. $0.1<D P[?] 0.3,0.3<D P[?] 0.5$ and $0.5<D P[?] 0.7 \mathrm{rep}$ $0.3<D P[?]-0.1,-0.5<D P[?]-0.3 a n d-0.7<D P[?]-0.5$ representedslight, moderateandseveredeclinesinhabitatsuitability, $r$

\section{Results}

\subsection{Model optimization}

Through different combinations of feature classes and regularized multipliers, the best model parameter is selected as among all combinations (feature classes: linear, product, threshold, and hinge; regularized multipliers $=2$ ), the parameters of all candidate models are shown in Table S3.

In the simulation of the current and future distribution of $B$. gargarizans, the AUC value indicated that the simulation results of all models reached an excellent level (0.9202[?] Training AUC[?]0.9306, 0.9001[?] Test AUC[?]0.9165, SD[?]0.014), all AUC values are presented in Table S4

\subsection{Current distribution}

After running maxent model for 10 times, the average AUC value was 0.926, Standard deviation is 0.01 (Fig.1).. The model validation results show that the model prediction results are excellent and can be used in the following analysis

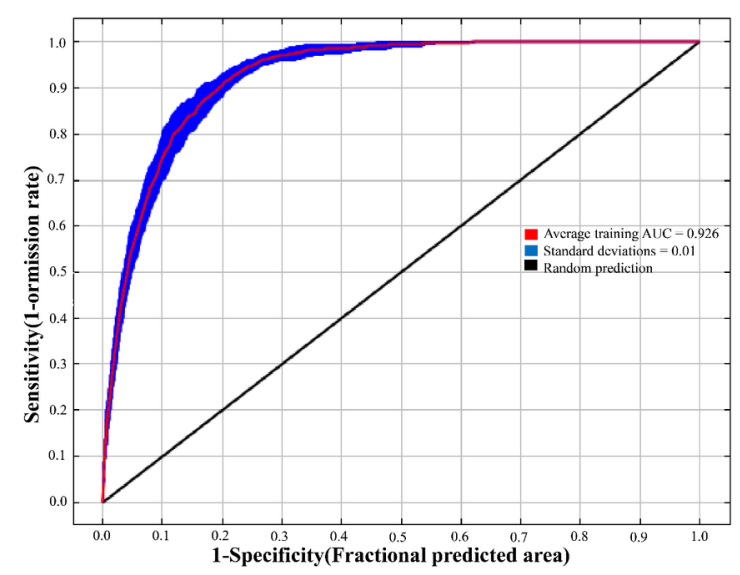

Fig. 1. Receiver operating characteristic (ROC) curve and average test AUC for accuracy analysis of Bufo gargarizan by MaxEnt model under the current situation. 
We found that the most suitable habitats were mainly in the midwest and the eastern coastal areas from current distribution of B. gargarizans(Fig.2). The threshold value of MSS method is 0.33 , and according to the distribution probability of B. gargarizans, China is divided into four grades: highly suitable habitat (HS, $\mathrm{P}[?] 0.75)$; medium suitable habitat $(\mathrm{MS}, 0.5[?] \mathrm{P}<0.75)$; poorly suitable habitat $(\mathrm{PS}$, threshold[?] $\mathrm{P}<0.5)$; unsuitable habitat(US, threshold $<\mathrm{P}[?] 0)$, and the areas of these four types of habitats were calculated in Arcgis, as shown in Table 1. Among them, the unsuitable habitat is $7,216,700$ square kilometers, accounting for 75.69 percent of Chinese areas.

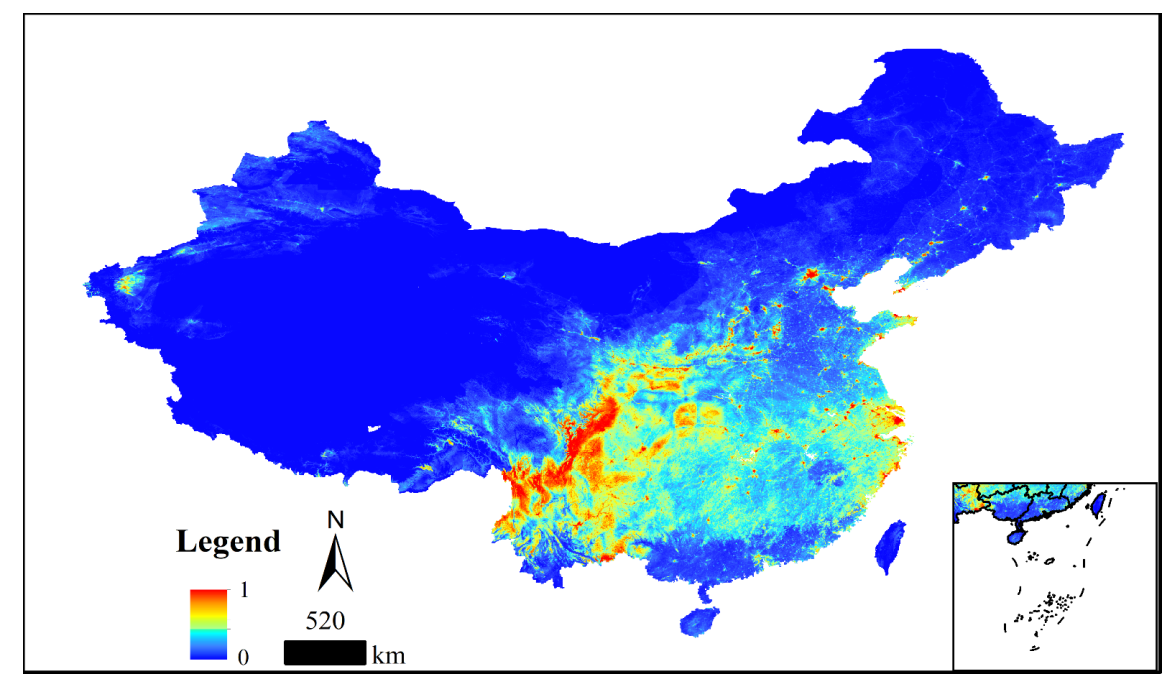

Fig.2 Current distribution of MaxEnt models for B. gargarizans in China. The color scale from blue to red indicates the habitat suitability value from 0 to 1.

Table1 Current habitat composition of $B$. gargarizans in China under MaxEnt model.HS: highly suitable habitat; MS: medium suitable habitat; PS: poorly suitable habitat;US: unsuitable habitat.

\begin{tabular}{llllllll}
\hline Species & Area $\backslash 10^{4} \mathrm{~km}^{2}$ & Area $\backslash 10^{4} \mathrm{~km}^{2}$ & Area $\backslash 10^{4} \mathrm{~km}^{2}$ & Area $\backslash 10^{4} \mathrm{~km}^{2}$ & Percentage $\backslash \%$ & Percentage $\backslash \%$ & Perce \\
\hline & US & PS & MS & HS & US & PS & MS \\
B. gargarizans & 721.67 & 134.16 & 67.63 & 30.35 & 75.69 & 14.07 & 7.1 \\
\hline
\end{tabular}

\subsection{Contribution of environmental variables}

The Jackknife analyses results showed that the variables with high contribution to the model were Bio9, Bio14, and POP, with a total contribution of $70.8 \%$. Among them, the contribution rate of Bio9 is $38.4 \%$, that of Bio14 and POP is $16.2 \%$, detailed analysis results are shown in the Table S5. Meanwhile, the range, mean value and optimal range of each variable (the probability of species existence $>0.5$ ) is shown in Table S6 and Fig.3. For variables with higher contribution (Bio9, Bio14, POP), there were significant differences in the mean values between the locations of occurrence and absence. 

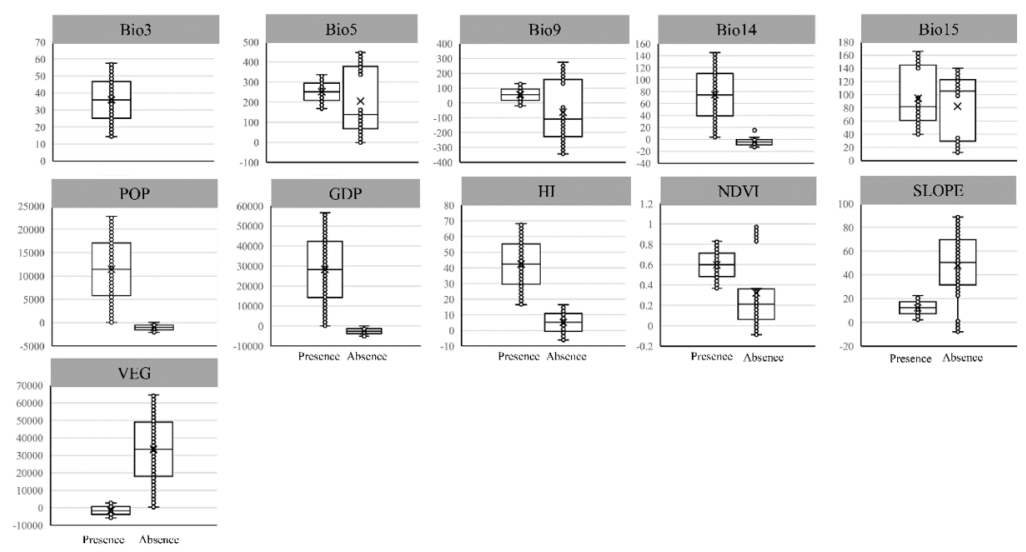

Fig.3 The distribution of predictor bioclimatic and topographic variables over the study area on presence and absence (background) locations.

\subsection{Analysis of future habitat changes}

The areas and proportions of the four habitats of B. gargarizansby 2050 and 2070 are shown in the Table S7. Compared with the current distribution, the future suitable habitat of B. gargarizans will be reduced in 2050 , and the ranges of highly suitable habitat, medium suitable habitat, poorly suitable habitat are $-1.3^{\sim}$ $0,-3.41 \sim-0.68$, and $-3.98 \sim 1.32$, and the mean values are $-0.72,-2.43$, and -1.42 respectively. The suitable habitat of B. gargarizans will shrink further in 2070, and the ranges of highly suitable habitat, medium suitable habitat, poorly suitable habitat are $-1.42 \sim-0.37,-3.71 \sim-1.59$, and $-3.94 \sim-0.03$, and the mean values are $-0.93,-2.71,-2.04$ respectively(Fig.4). And the overall change trend and degree of suitable habitats of B. gargarizans in China is shown in Fig.5 and Table S8. In addition, the potential distribution area of Chinese toad in the future shows a trend of fragmentation, which is gradually serious over time, and this trend is related to RCPs to a certain extent (RCPS8.5 is the most serious)(Fig. S1)

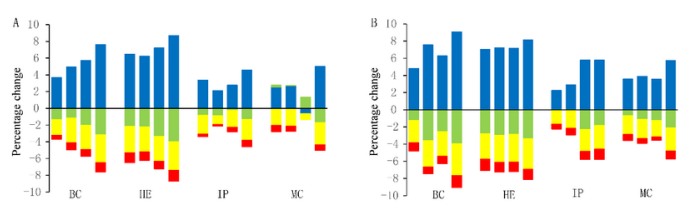

Fig.4 The four levels of suitable habitat fluctuation ranges of $B$. gargarizans under different future climate scenarios in China. Red, orange, green, and blue represent increases (positive number) or decreases (negative number) of the proportion of highly suitable, medium 
suitable, poorly suitable, and unsuitable habitat, respectively. BC, HE, IPand MC represent BCC-CSM1-1, HadGEM2-ES, IPSL-CM5A-LR and MIROC5, respectively, which are different global climate models. $A$ is in $2050, B$ is in 2070.

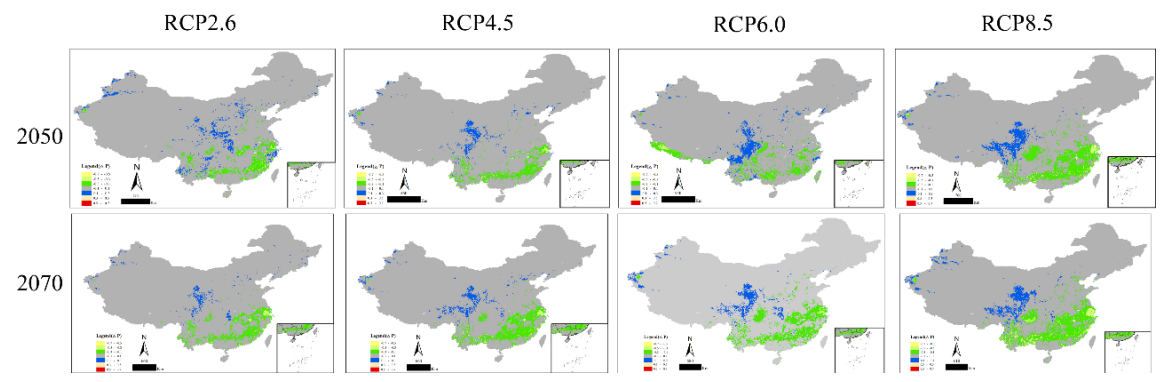

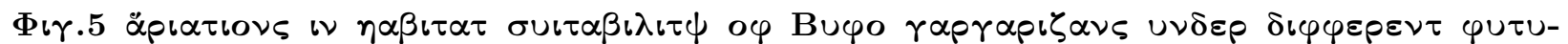

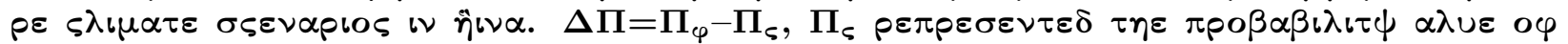

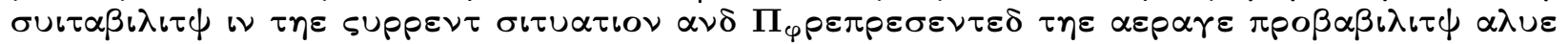

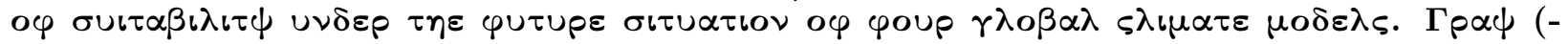

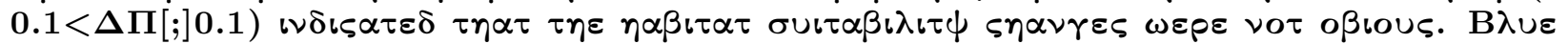

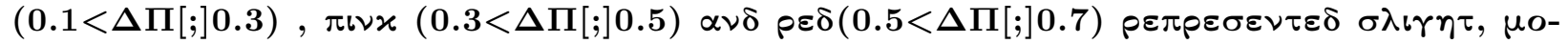

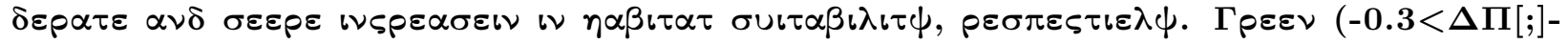

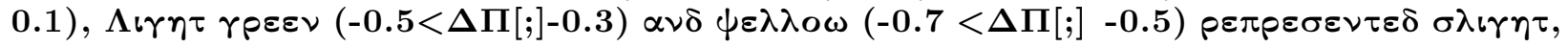

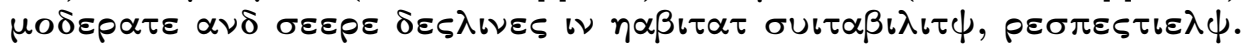

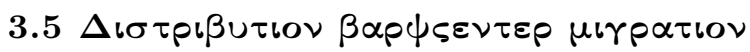

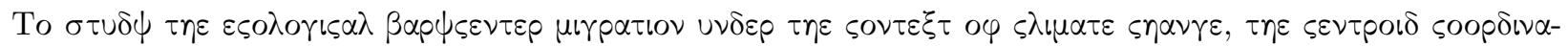

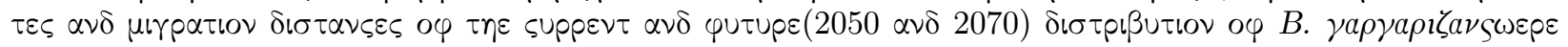

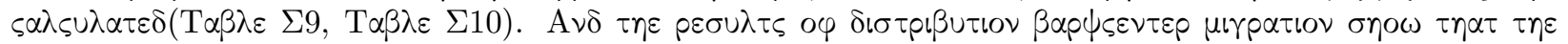

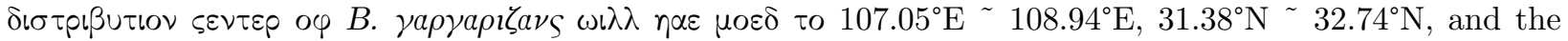
distance of barycenter migration will reach $38.47 \mathrm{~km} \sim 249.45 \mathrm{~km}$. And by 2070 , the distribution of B. gargarizans will continue to change, and its distribution center will migrate to $107.00^{\circ} \mathrm{E} \sim 108.75^{\circ} \mathrm{E}, 31.95^{\circ} \mathrm{N} \sim$ $32.37^{\circ} \mathrm{E}$, the migration distance is between $93.15 \mathrm{Km}$ and $270.67 \mathrm{Km}$. Fig. 5 and Fig. 6 show that the suitable distribution area of B. gargarizans will decrease in the southeast, and gradually migrate and increase to the midwest in China.

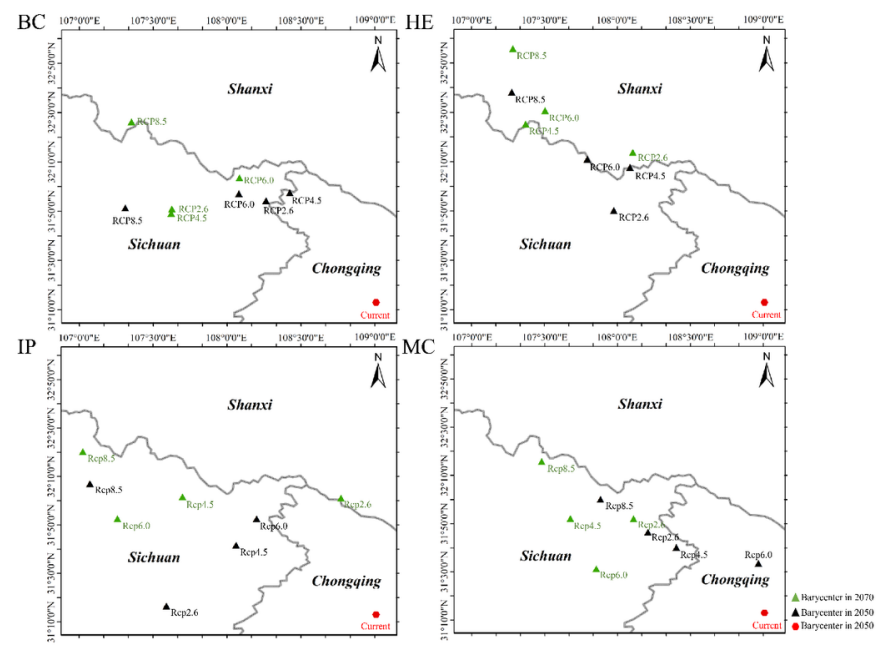


Fig. 6 Barycenter transfer of Bufo gargarizans in China. The red hexagon represents the barycenter of a suitable habitat under current scenarios. The black and green triangles represent the barycenter of suitable habitats in 2050 and 2070, respectively, using four global climate models.

\section{Discussion}

\subsection{Maxent model and environmental variables}

Maxent model can connect the distribution data of species with geographic and ecological information, and calculate the maximum entropy value of species distribution, so as to predict the potential distribution area of species(Giannakopoulos et al., 2019). Maxent model can achieve a good prediction effect only by using limited species distribution data. Therefore, it is often used to simulate the potential distribution area of species, drift of potential distribution area, suitability evaluation and other studies on global climate change involving species distribution and genetic diversity in conservation biology and invasion biology(Hannah et al., 2007; Mays, Hung, Shaner, Denvir, \& Primerano, 2018; Prieto-Torres, Navarro-Sigüenza, SantiagoAlarcon, \& Rojas-Soto, 2016).

Environmental variables commonly used for Maxent model analysis include climate variables, landscape variables such as topography and landform, and the impact of human factors on species distribution is rarely considered (Pearson, Raxworthy, Nakamura, \& Townsend, 2010; Penman, Pike, Webb, \& Shine, 2010). However, the influence of human factors on ecological environment is extensive and continuous, and its role cannot be ignored(Li et al., 2014). From the contribution of the four environmental factors selected in this study, the climate factor is still the main factor affecting distribution of B. gargarizans in China. Among them, the contribution of Bio9 and Bio14 is relatively large, the contribution was $38.4 \%$ and $16.2 \%$, and the optimal distribution range of B. gargarizans was $-1.85 \sim 13.01$ and $3.59 \mathrm{~mm} \sim 145.20 \mathrm{~mm}$, respectively. Secondly, human influence also has a certain degree of contribution, and the overall influence trend shows that, within a certain range, the distribution probability of B. gargarizans increases with the enhancement of human influence (Table S5, Fig.3), this is caused by the overlap of the ecological niche of the B. gargarizans and the area of human activities and some human infrastructure is more conducive to the distribution of amphibians(Tytar, Sobolenko, Nekrasova, \& Mezhzherin, 2015). Compared with other environmental factors, the overall contribution of topographic and geomorphic factors is relatively low, and see Fig.7 for details.

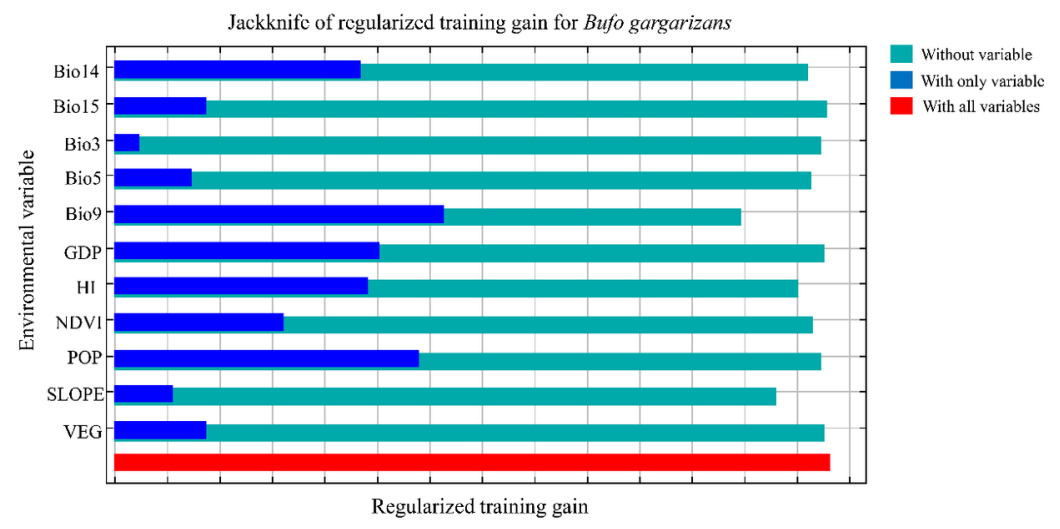

Fig.7 Results of jackknife train of relative importance of predictor variables forB. gargarizans 4.2 Future distribution change and barycenter migration ofB. gargarizans 
Many studies suggest that global temperatures are rising and will continue to do so for some time to come, with an expected increase of 0.6 in the 20th century and $1.4 \sim 5$ by the 21th century (Budikova, 2009; Mccarthy, Canziani, Leary, Dokken, \& White, 2007). The rising global temperature will inevitably affect the change of species distribution, which will eventually lead to two results: one is the reduction of species distribution, which will eventually lead to extinction of species; the other is the migration of species to previously undistributed areas, which will expand the distribution (Bellard, Bertelsmeier, Leadley, Thuiller, \& Courchamp, 2012; Morueta-Holme, Camilla, \& Svenning, 2010; Thomas et al., 2004).

In this study, under the background of climate change, the suitable distribution area of B. gargarizans will continue to decrease in the next $30^{\sim} 50$ years. In comparison to three other global Change Scenarios, we can see the smallest reduction in suitable habitat for B. gargarizans under RCP2.6 Scenarios (The most optimistic scenario is that greenhouse gas emissions peak between 2010 and 2020, then continue to decline, with a negative value by the end of the century). And the suitable habitat of $B$. gargarizans will be further reduced Under the scenarios of RCP4.5 and RCP6.0(That represents a decline after greenhouse gas emissions peaked in 2040 and 2080, respectively). the suitable habitat of B. gargarizans of China decreased to the maximum extent under the condition of RCP8.5(Greenhouse gas emissions continue to rise in this century). The results show that with the increase of greenhouse gas emission concentration, the area of suitable habitat of $B$. gargarizans decreases more, on the other hand, it suggests the importance of energy conservation and emission reduction to the maintenance of ecological balance.

As show in Fig. 4 and Fig. S5, the suitable habitat of B. gargarizans will decrease in the south, southeast coast and central part of China, and correspondingly increase in the central and western part of China from now to 2050. And the trend will become more pronounced by 2070. In the next 30 to 50 years, the distribution centers of B. gargarizanswill gradually move from Yunyang County in northeast Chongqing City to Wanyuan County, Tongjiang County and Xuanhan County in northeast Sichuan Province and Langao County, Ziyang County, Zhenba County, Nanzheng County and Chenggu County in southwest Shaanxi Province. Compared with the present, the distribution focus has gradually shifted to the northwest, and this trend has not weakened with time. In addition, it can be seen from Figure 1 that Bio9 and Bio14, two climatic factors that have a great influence on the distribution of B. gargarizans . are increasing from 2050 to 2070 compared with the present, and the growth trend is more obvious in eastern and southern China. And these are the areas where B. gargarizans, will be less concentrated in the future. And the reason for this phenomenon is that the average temperature and precipitation in these regions are higher at present, and the further increase of temperature and precipitation in the future will make the values of these factors beyond the range suitable for the distribution of B. gargarizans (Table S6) .

Four GCMs under four RCPs were used in this study, and the results show that under the global climate change, like other species that have been reported, the distribution area of B. gargarizans will migrate to the high latitude and high altitude area (China's terrain is low in the east)(Kelly \& Goulden, 2008; Parmesan \& Yohe, 2003). On the other hand, this paper is more inclined to think that the current distribution pattern of B. gargarizans, is caused by the dispersion, because from the perspective of the current distribution, there is no obvious geographical isolation between the distribution areas of different regions of B. gargarizans ," and the future distribution of $B$. gargarizans, showed that the future distribution of B. gargarizans, would gradually break up, and the geographical separation would gradually form between different regions (Fig.2, Fig. S1). The results of this study indicated that the distribution area of B. gargarizans would be reduced in the future, and the habitat connectivity, resilience and gene exchange between the populations would be reduced with the serious habitat fragmentation year by year, finally leading to the gradual decline of $B$. gargarizans population(Duan et al., 2016). And it suggests that some amphibians, which are more sensitive to environmental changes and narrower than $B$. gargarizans, the changes to their niches from climate change will be even more dramatic and even devastating. Due to the influence of the resolution of the environmental layer, the prediction results cannot be very accurate. However, we still believe that this study will provide theoretical support for the direction of conservation and ecological management of B. gargarizans, and it is believed that it can provide a research direction for other amphibians to cope with climate change. 


\section{Data Accessibility:}

Occurrence records of B. gargarizans were obtained from laboratory survey and global biodiversity information platform (GBIF), and the data finally used for analysis are listed in Table S1 in the attachment.

Environment variables are derived from official websites such as Worldclim, Resource and Environment Science and Data Center and Last of the Wild Data. Please refer to the Table S2 in the attachment for the specific download sites.

\section{References}

Bellard, C., Bertelsmeier, C., Leadley, P., Thuiller, W., \& Courchamp, F. (2012). Impacts of climate change on the future of biodiversity. Ecology Letters, 15(4), 365-377.

Bertrand, R., Lenoir, J., Piedallu, C., Riofrio-Dillon, G., Ruffray, P., Vidal, C., Gegout, J. C. (2011). Changes in plant community composition lag behind climate warming in lowland forests. Nature, 479, 517-520.

Budikova, D. (2009). Role of Arctic sea ice in global atmospheric circulation: A review. Global Planetary Change, 68(3), 149-163.

Cobos, M. E., Peterson, A. T., Barve, N., \& Osorio-Olvera, L. (2019). Kuenm: An R package for detailed development of ecological niche models using Maxent. PeerJ, 7(1), e6281.

Dawson, T. P., Jackson, S. T., House, J. I., Prentice, I. C., \& Mace, G. M. (2011). Beyond redictions: Biodiversity conservation in a changing climate. 332(6025), 53-58.

Duan, R. Y., Kong, X. Q., Huang, M. Y., Varela, S., \& Ji, X. (2016). The potential effects of climate change on amphibian distribution, range fragmentation and turnover in China. PeerJ, 4(10), e2185.

Elith, J., Leathwick, J. R. (2009). Species Distribution Models: Ecological Explanation and Prediction Across Space and Time. Annual Review of Ecology Evolution \& Systematics, 40(1), 677-697.

Fu, J. Z., Weadick, C. J., Zeng, X. M., Wang, Y. Z., \& Hu, Y. (2005). Phylogeographic analysis of the Bufo gargarizans species complex: A revisit. Molecular PhylogeneticsEvolution, 37(1), 202-213.

Giannakopoulos, A., Vasileiou, N. G. C., Gougoulis, D. A., Cripps, P. J., Ioannidi, K. S., \& Chatzopoulos, D. C. (2019). Use of geographical information system and ecological niche modelling for predicting potential space distribution of subclinical mastitis in ewes. Veterinary microbiology, 228, 119-129.

Guisan, A., \& Thuiller, W. (2010). Predicting species distribution: offering more than simple habitat models. Ecology Letters, 8(9), 993-1009.

Hannah, L., Midgley, G., Andelman, S., Araujo, M., Hughes, G., Martinez-Meyer, E., . . . Williams, P. (2007). Protected area needs in a changing climate. Front. Ecol. Environ., 5, 131-138.

He, Y. B., Chen, Y. Q., Tang, H. J., Yao, Y. M., Yang, P., \& Chen, Z. X. (2011). Exploring spatial change and gravity center movement for ecosystem services value using a spatially explicit ecosystem services value index and gravity model. Environmental Monitoring Assessment, 175, 563-571.

Hijmans, R. J., Phillips, S., Leathwick, J., \& Elith, J. (2017). Dismo: species distribution modeling. R Package Version, 1(4), 1-1.

Hu, Y. L., Wu, X. B., Jiang, Z. G., Yan, P., Su, X., \& Cao, S. Y. (2007). Population Genetics and Phylogeography of Bufo gargarizans in China. Biochemical Genetics, 45, 697-711.

Jiang, F., Zhang, J. J., Gao, H. M., Cai, Z. Y., \& Zhang, T. Z. (2019). Musk deer (Moschus spp.) face redistribution to higher elevations and latitudes under climate change in China. Science of The Total Environment, 704, 135335.

Kelly, A. E., \& Goulden, M. L. (2008). Rapid shifts in plant distribution with recent climate change. Proceedings of the National Academy of ences of the United States of America, 105(33), 11823-11826. 
Lambers, J. H. R. (2015). Ecology. Extinction risks from climate change. Science, 348, 501-502.

Li, R., Xu, M., Wong, M. H. G., Qiu, S., Sheng, Q., Li, X., \& Song, Z. (2014). Climate change-induced decline in bamboo habitats and species diversity: implications for giant panda conservation Diversity \& Distributions, 21(4), 379-391.

Liu, C., White, M., Newell, G., \& Pearson, R. (2013). Selecting thresholds for the prediction of species occurrence with presence-only data. Journal of Biogeography, 40(4), 778-789.

Mays, H. L., Hung, C. M., Shaner, P. J., Denvir, J., \& Primerano, D. A. (2018). Genomic Analysis of Demographic History and Ecological Niche Modeling in the Endangered Sumatran Rhinoceros Dicerorhinus sumatrensis. Current Biology, 28(1), 70-76.

Mccarthy, J. J., Canziani, O. F., Leary, N. A., Dokken, D. J., \& White, K. S. (2007). Climate Change 2001: Impacts, Adaptation and Vulnerability - Contribution of Working Group II to the Third Assessment Report of IPCC. Contribution of Working Group II to the Third Assessment Report, 19(2), 81-111.

Morueta-Holme, N., Camilla, F., \& Svenning, J. C. (2010). Climate change risks and conservation implications for a threatened small-range mammal species. PLoS ONE, 5(4), e10360.

Nogues-Bravo, D., \& Rahbek, C. (2011). Communities Under Climate Change. Science in China Series C: Life Sciences, 334, 1070-1071.

Parmesan, C., \& Yohe, G. (2003). A globally coherent fingerprint of climate change impacts across natural systems. Nature, 421, 37-42.

Pearson, R. G., \& Dawson, T. P. (2003). Predicting the impacts of climate change on the distribution of species: are bioclimate envelope models useful? Global Ecology Biogeography, 12, 361-371.

Pearson, R. G., Raxworthy, C. J., Nakamura, M., \& Townsend, P. A. (2010). ORIGINAL ARTICLE: Predicting species distributions from small numbers of occurrence records: a test case using cryptic geckos in Madagascar. Journal of Biogeography, 34(1), 102-117.

Penman, T. D., Pike, D. A., Webb, J. K., \& Shine, R. (2010). Predicting the impact of climate change on Australia's most endangered snake, Hoplocephalus bungaroides. Diversity \& Distributions, 16(1), 109-118.

Preston, K. L., Rotenberry, J. T., Redak, R. A., \& Allen, M. F. (2010). Habitat shifts of endangered species under altered climate conditions: importance of biotic interactions. Global Change Biology, 14(11), 2501-2515.

Prieto-Torres, D. A., Navarro-Sigüenza, A. G., Santiago-Alarcon, D., \& Rojas-Soto, O. R. (2016). Response of the endangered tropical dry forests to climate change and the role of Mexican Protected Areas for their conservation. Global Change Biology, 22(1), 364-379.

Sinervo, B., Mendez-de-la-Cruz, F., Miles, D. B., Heulin, B., Bastiaans, E., \& Cruz, M. V. S. (2010). Erosion of Lizard Diversity by Climate Change and Altered Thermal Niches. \%J Science. Science, 328, 894-899.

Stuart, S. N., Chanson, J. S., Cox, N. A., Young, B. E., Rodrigues, A. S. L., Fischman, D. L., \& Waller, R. W. (2004). Status and Trends of Amphibian Declines and Extinctions Worldwide. Science, 306(5702), 1783-1786.

Thomas, C. D., Cameron, A., Green, R. E., Bakkenes, M., Beaumont, L. J., Collingham, Y. C., . . . Hannah, L. (2004). Extinction risk from climate change. Nature, 427(6970), 145-148.

Tytar, V., Sobolenko, L., Nekrasova, O., \& Mezhzherin, S. (2015). Using Ecological Niche Modeling For Biodiversity Conservation Guidance In The Western Podillya (Ukraine): Amphibians. Vestnik Zoologii, $49(6), 551-585$. 
Wake, D. B., \& Vredenburg, V. T. (2008). Are we in the midst of the sixth mass extinction? A view from the world of amphibians. Proceedings of the National Academy of Sciences of the United States of America, 64(1), 11466-11473.

Yang, C., Tang, S. H., \& Luo, Z. H. (2020). Distribution Changes of Chinese Skink(Eumeces chinensis) in China: the Impacts of Global Climate Change. Asian Herpetological Research, 11(2), 132-138. 